\title{
Herramientas de gestión para la evaluación de estrategias en los cuerpos de bomberos.
}

\section{Management tools for the evaluation of strategies in the fire departments.}

\author{
Mirka Lissette Montoya Santillán. ${ }^{1}$, Katherine Janeth Masaquiza Sailema. ${ }^{2}$, María \\ Teresa Espinosa Jaramillo. ${ }^{3} \&$ Mayorga Díaz Mónica Patricia. ${ }^{4}$
}

DOI: https://doi.org/10.33262/visionariodigital.v5i2.1637

\begin{abstract}
Within the National Development Plan 2017-2021 Ecuador establishes its axis 2 called economy at the service of society, where it defines national objective 5: boost productivity and competitiveness for economic and sustainable growth in a redistributive and supportive manner; which shows a close relationship with the objective of this research, where the cost control of production processes, optimization of resources, strategic actions that allow the improvement of economic administration in entities called Fire Brigades, which are analyzed they find settled throughout the world with the purpose of preventing, protecting and helping the community. In developed countries, fire departments have greater accessibility to equipment, training for different operational areas; In Ecuador, the fire brigades with the greatest equipment provision are for the stations of the cities with the highest population and vulnerability of risks at the national level, and also have more access to training for personnel. Therefore, it is necessary to carefully analyze the application of management tools that allow the evaluation of strategies, which are in accordance with the current legal framework.
\end{abstract}

\footnotetext{
1 Ingeniera en Contabilidad y Auditoría, profesional independiente, Correo electrónico: mirkaamontoyaa@gmail.com, https://orcid.org/0000-0001-5008-2508

2 Licenciada en Contabilidad y Auditoría, profesional independiente, katherinejms96@gmail.com, https://orcid.org/0000-0002-1616-7337

3 Magíster en auditoría integral, Universidad Arturo Prat del Estado de Chile, espinosa.auditores@gmail.com,https://orcid.org/0000-0002-6006-3826

4 Docente investigadora, Doctora en Ciencias Económicas, Universidad Regional Autónoma de Los Andes, Facultad de Dirección de Empresas, Carrera de Contabilidad y Auditoría. monikmayorgad@gmail.com, https://orcid.org/0000-0003-3071-236X
} 
Keywords: process, review, corrective actions, community, service

\section{Resumen}

Dentro del Plan Nacional de Desarrollo 2017 - 2021 el Ecuador establece su eje 2 denominado economía al servicio de la sociedad, en donde define el objetivo nacional 5; lo cual muestra estrecha relación con el objetivo de esta investigación, en donde se analiza el control de costos de procesos de producción, optimización de recursos, acciones estratégicas que permitan el mejoramiento de la administración económica en las entidades llamadas Cuerpos de Bomberos, la cuales se encuentran asentadas a lo largo del mundo con la finalidad de prevenir, proteger y socorrer a la comunidad. En los países desarrollados los cuerpos de bomberos tienen mayor accesibilidad a equipamiento, capacitaciones para las diferentes áreas operativas; los cuerpos de bomberos en el Ecuador con mayor dotación de equipamiento son para las estaciones de las ciudades con mayor población y vulnerabilidad de riesgos a nivel nacional, e igual manera tienen más acceso a capacitaciones para el personal. Por consiguiente, es necesario analizar minuciosamente la aplicación de herramientas de gestión que permitan la evaluación de estrategias, que estén acorde al marco jurídico vigente.

Palabras claves: proceso, revisión, acciones correctivas, comunidad, servicio.

\section{Introducción}

Los Cuerpos de Bomberos se originan en los inicios de la humanidad, podríamos decir estar seguros que el descuido nació en el hombre antes de la idea de frotar pedernales para hacer fuego con que cocinar sus alimentos y calentarse los fríos días invernales. (Cuerpo de bomberos voluntaio amaga, 2018) En la historia se indica que debe estar compuesto por esclavos, pasando por la obligación bajo multa, hasta llegar al sentido social y cívico de los actuales Cuerpos de Bomberos. Todo un camino recorrido en más de 2000 años de vigencia al servicio de la Comunidad. (Casanova, 2010) En el mundo los bomberos ayudan a proteger a la comunidad, asesorando e informando a los ciudadanos acerca de la seguridad y prevención de incendios. Se les llama a luchar contra los incendios y también frente a las emergencias, tales como accidentes de carretera y ferrocarril, y en inundaciones, el trabajo implica el uso de una amplia gama de equipamiento. (Educaweb, s.f.)

En el Ecuador los Cuerpos de Bomberos son entidades de derecho público adscritas a los Gobiernos Autónomos Descentralizados (GAD) municipales o metropolitanos, que prestan el servicio de prevención, protección, socorro y extinción de incendios, así como de apoyo en otros eventos adversos de origen natural o antrópico. Así mismo efectúan acciones de salvamento con el propósito de precautelar la seguridad de la ciudadanía en su respectiva circunscripción territorial. (Código Orgánico de Organización Territorial, Autonomía y Descentralización COOTAD, 2010) 
Las estaciones de los Cuerpos de bomberos se encuentran ubicadas según la cantidad poblacional, situación geográfica y normas nacionales e internacionales específicamente existente en las cabeceras cantonales hoy en día existen 160 cuerpos de bomberos en el Ecuador, de ellos tan solo 44 se han descentralizados a los GAD municipales, los mismos que están prestos para el servicio a la ciudadanía, destinados específicamente a defender las propiedades contra el fuego, socorrer en catástrofes o siniestros a las personas.

Los bomberos son empleados de la Administración Pública, se regirán a la ley orgánica deservidores públicos y al código de trabajo con respecto a la dependencia laboral y en el accionar de sus funciones, esta profesión no es ni cómoda ni fácil; se trata de un trabajo que expone al individuo a un elevado nivel de estrés y peligro. La profesión de bombero requiere un grado alto de dedicación personal, un verdadero deseo de ayudar a la gente y un gran nivel de pericia. (Secretaría de gestión de riesgo, 2016) (Secretaría de gestión de riesgo, 2017)

En el cantón Shushufindi es una entidad de derecho público, adscrito al GAD Municipal del Cantón Shushufindi, mediante ordenanza que regula la gestión de los servicios de prevención, protección, socorro y extinción de incendios en el cantón, publicada en el registro oficial año III-N580, de 19 de mayo del 2016. Como entidad de primera respuesta fundamenta su propósito en la provisión oportuna y eficiente de servicios de prevención, protección, socorro, extinción de incendios, además de asistencia en desastres y emergencias, utilizando protocolos de operación normalizados, con equipamiento adecuado para tranquilidad y satisfacción de la comunidad, tanto en las áreas operativas como administrativas, dentro de los parámetros de calidad y calidez (Gobierno Autónomo Descentralizado Municipal de Shushufindi, 2016)

Los Cuerpos de Bomberos en cada parte del mundo tienen el mismo objetivo prevenir proteger y socorrer a la comunidad.

En los países desarrollados los cuerpos de bomberos tienen mayor accesibilidad a equipamiento, capacitaciones para las diferentes áreas operativas; los cuerpos de bomberos del Ecuador con mayor dotación de equipamiento son para las estaciones de las ciudades con mayor población y vulnerabilidad de riesgos a nivel nacional, e igual manera tienen más acceso a capacitaciones para el personal. En las demás estaciones son dotadas con menos cantidad de equipamiento y es de menos facilidad acceder a las capacitaciones, razones por las cuales no les permite cumplir al máximo sus objetivos y metas propuestas. Para lo cual es necesario analizar minuciosamente la aplicación de herramientas de gestión que permita la evaluación de estrategia, que estén acorde al marco jurídico vigente (Tabla 1.). 
Tabla 1. Normativa Jurídica

Normativa

(Consititucion del

Ecuador, 2008)

Registro Oficial 449 de 20 de octubre de 2008

\section{Descripción}

Art. 11.- EI ejercicio de los derechos se regirá por los siguientes principios:

3). Los derechos y garantías establecidos en la Constitución $\mathrm{y}$ en los instrumentos internacionales de derechos humanos serán de directa e inmediata aplicación por y ante cualquier servidora o servidor público, administrativo o judicial, de oficio o a petición de parte. Para el ejercicio de los derechos y las garantías constitucionales no se exigirán condiciones o requisitos que no estén establecidos en la Constitución o la ley. Los derechos serán plenamente justiciables. No podrá alegarse falta de norma jurídica para justificar su violación o desconocimiento, para desechar la acción por esos hechos ni para negar su reconocimiento. (Consititucion del Ecuador, 2008)

Art. 226.- Las instituciones del Estado, sus organismos, dependencias, las servidoras o servidores públicos y las personas que actúen en virtud de una potestad estatal ejercerá solamente las competencias y facultades que les sean atribuidas en la Constitución y la ley. Tendrán el deber de coordinar acciones para el cumplimiento de sus fines y hacer efectivo el goce y ejercicio de los derechos reconocidos en la Constitución. (Consititucion del Ecuador, 2008)

(Consejo de

Participación

Ciudadana y Control

Social, 2004) Ley

Orgánica de

Transparencia y Acceso

a la Información

Pública LOTAIP

Registro Oficial 337 de

Art. 7 Difusión de la Información Pública.- a) Estructura orgánica funcional, base legal que la rige, regulaciones y procedimientos internos aplicables a la entidad; las metas y objetivos de las unidades administrativas de conformidad con sus programas operativos; h) Los resultados de las auditorías internas y gubernamentales al ejercicio presupuestal; k) Planes y programas de la institución en ejecución; (Consejo de Participación Ciudadana y Control Social, 2004)

18-mayo-2004

(Código Orgánico de Art. 3.- Principios. - El ejercicio de la autoridad y las Organización potestades públicas de los gobiernos autónomos 
Territorial, Autonomía descentralizados se regirán por los siguientes principios: “ y Descentralización ... b) Solidaridad. - Todos los niveles de gobierno tienen COOTAD, 2010)

Registro Oficial 303 de 19-octubre-2010 como obligación compartida la construcción del desarrollo justo, equilibrado y equitativo de las distintas circunscripciones territoriales, en el marco del respeto de la diversidad y el ejercicio pleno de los derechos individuales y colectivos. En virtud de este principio es deber del Estado, en todos los niveles de gobierno, redistribuir y reorientar los recursos y bienes públicos para compensar las inequidades entre circunscripciones territoriales; garantizar la inclusión, la satisfacción de las necesidades básicas y el cumplimiento del objetivo del buen vivir..." (Código Orgánico de Organización Territorial, Autonomía y Descentralización COOTAD, 2010)

(Código Orgánico de las Entidades de

Seguridad Ciudadana y Orden Público

COESCOP, 2017)

Registro Oficial 19 de 21-junio-2017
Art. 276.- Funciones. - “... 3). Estructurar y ejecutar campañas de prevención y control de desastres naturales o emergencias, ¿orientadas a la reducción de riesgos en coordinación con el ente rector nacional; 4). Diseñar y ejecutar planes y programas de capacitación para prevenir y mitigar los efectos de desastres naturales y emergencias en coordinación con los Gobiernos Autónomos Descentralizados metropolitanos o municipales y con el ente rector nacional de gestión de riesgos. . (Código Orgánico de las Entidades de Seguridad Ciudadana y Orden Público COESCOP, 2017)

Art. 285.- Modelo de Gestión Cantonal. Los Cuerpos de Bomberos elaborarán su modelo de gestión de acuerdo a la categorización de la autoridad responsable de la planificación nacional y la autoridad rectora en gestión de riesgos. (Código Orgánico de las Entidades de Seguridad Ciudadana y Orden Público COESCOP, 2017)

(Gobierno Autónomo Art. 32.- Funciones del Primer Jefe. - “ ... 9). Elaborar los

Descentralizado

Municipal

Shushufindi, 2016)

Registro Oficial 580 de 19-mayo-2016
Reglamentos, el Plan Operativo Anual y la Proforma de Presupuestaria y darle trámite legal correspondiente. 12). Presentar al Consejo de Administración y Disciplina los informes económicos, balances, informes operativos y de gestión anual ..." (Gobierno Autónomo Descentralizado Municipal de Shushufindi, 2016) 
(Plan Nacional de Objetivo 8. Promover la transparencia y la Desarrollo, 2017) corresponsabilidad para una nueva ética social; 8.2.) SENPLADES Fortalecer la transparencia en la gestión de instituciones públicas y privadas y la lucha contra la corrupción, con 2017-2021 mejor difusión y acceso a información pública de calidad, optimizando las políticas de rendición de cuentas y promoviendo la participación y el control social. (Plan Nacional de Desarrollo, 2017)

Elaboración propia a partir de la bibliografía consultada

La gestión es un conjunto de herramientas claves de acciones, operaciones y administración de la alta dirección de una organización alineada a las políticas, valores de organización, recursos para la productividad y rendimiento, buscando el mejoramiento continuo durante el proceso de planeación, implantación, y control de estrategias de procesos, teniendo así la capacidad de administrar eficazmente los flujos del conocimiento para una buena toma de decisiones y poder alcanzar objetivos y metas. (Bolaños, 2016); (Gonzáles \& Tapia, 2016); (Angulo, 2017); (Mora, Duran, \& Zambrano, 2016); (Medina, Medina, \& Nogueira, 2017).

Así como también constituye una de las columnas fundamentales de las instituciones para sostenerse y mantenerse mucho tiempo, porque permite generar estrategias en todas las áreas de la organización. Para un debido control de gestión la institución es importante tomar en cuenta todos los componentes que forman una institución los cuales son: administrativo, técnico, legal, internacional, contables y financiero, para que luego de ello si poder establecer objetivos estratégicos, también elaborar y aprobar un plan, en el cual se plantee responsabilidades a las personas para que puedan cumplir con los asignando y presupuesto anual, con esto aplicar medidas correctivas si en el seguimiento pudiera detectar algún inconveniente, para esto es necesario que se reúnan mínimo una vez por año todos los miembros de la institución （Carvajal \& Escobar, 2013) (Pereira, 2019)

Las herramientas de gestión, ayudan a una organización en los aspectos de generales para el registro de datos, consolidación de datos, toma de decisiones y para rediseñar los procesos, que sirve para medir el cumplimiento de los objetivos a su vez proporciona metodologías por la dirección que facilita el control de operaciones; fundamenta el conocimiento de activos intangibles, las cuales sirven para evaluar las competencias personales, así canaliza habilidades y conocimientos específicos. Facilita los procesos internos, operaciones y comerciales, en la actualidad una herramienta importante parte de gerencia que abarca a todos los integrantes de a institución, así como también es el uso de las tecnologías de la información. (Mendez, 2009); (Velazco, 2009); (Vargas \& Aldana, 2014); (Hernandes \& Escobar, 2017); (Cuatrecasa \& Gonzales, 2017). 
Para que la gestión se operacionalice es menester plantear objetivos que hagan referencia a las actividades que realiza la organización, la cual permite evaluar y verificar la eficacia y eficiencia de las acciones realizadas para alcanzar las metas propuestas. Todo objetivo planteado debe ser cumplido de acuerdo al tiempo establecido y estipulado ya sea corto, mediano y largo plazo; una vez llegado al tiempo límite o mediante el proceso se realiza la evaluación de estrategia es decir la manera en la que se logró cumplir los objetivos. (Hernandez, 2014); (Palma \& Burgos, 2016); (Mora, Duran, \& Zambrano, 2016).

Por consiguiente el cumplimiento de objetivos debemos tomar en cuenta de que tipo de objetivos es el que queremos cumplir en nuestra institución, ya que depende mucho del tipo para poder elaborar y poner en practica estrategias y políticas que permitan un adecuado cumplimiento, evitando de tal manera reducir cualquier inconveniente que se produzca en el camino que impida el cumplimiento del mismo. Dentro de los tipos de objetivos tenemos: los objetivos operacionales los cuales se fijan solo en las operaciones que se realizan en la institución; objetivos de desempeño e información financiera, que básicamente se fijan en toda el área financiera de la institución; objetivo de cumplimiento se basa en que todo lo planteado este de acuerdo a la normativa legal; objetivo administrativos el cual se centra en la función administrativa. (Villamizar, 2011); (Henao, y otros, 2016); (Aranda, Ordoñez, \& Peralta, 2018)

El análisis y la definición de objetivos de una empresa permite y facilita el conocimiento de las debilidades y fortalezas que la afectan, tal hecho posibilita, así mismo, la definición de las estrategias administrativas que se deberán implementar para la adecuada gestión de los recursos empresariales, así como de los distintos factores de éxito y de riegos (Villamizar, 2011); también para el cumplimiento de objetivos depende del Estado, y gracias a la transformación como un ente basado con un enfoque moderno en la eficacia del cumplimiento de los objetivos, se ha podido lograr cumplir de una madera más eficaz; cada objetivo exige el diseño de instrumentos que ayuden a cumplir cada uno de ellos. (Henao, y otros, 2016); (Aranda, Ordoñez, \& Peralta, 2018).

Para una adecuada evaluación de estrategia se debe obtener toda información resultado del direccionamiento estratégico, que permitirá enfocar un criterio acerca de la gestión que está realizando la institución, debe estar basada en: la necesidad que permite reconocer si la planificación realizada va de acuerdo a las necesidades de la institución; efectividad es decir si se está realizando lo correcto con los recursos necesarios; comparabilidad donde se compara los logros alcanzados con los de los años anteriores, en consecuencia es un proceso importante que se lo debe planear desde un inicio, ya que esta permite conocer el desempeño no solo de la institución si también de quienes lo integran, para que al paso del tiempo se pueda realizar correcciones en el caso de que hubiera algún contratiempo, y si es necesario plantear nuevas estrategias si fuera necesario para cumplir con lo planeado. (Cruz, 2003) 
Además, es una fase de la administración estratégica, dirigida por los de la alta gerencia para evaluar la manera por la cual alcanzaron los objetivos. Las organizaciones sin importar la función económica a la que se dedica o de su dimensión deben aplicar el proceso de evaluar y revisar las estrategias con las cuales se cumplió los objetivos, lo cual es recomendable realizar de manera continúa estando al mando la alta dirección. (Chiavenato \& Sapiro, 2010); (Opazo, Sépulveda, \& Perez, 2015); (Fred \& Forest, 2017). (Figura 1.).

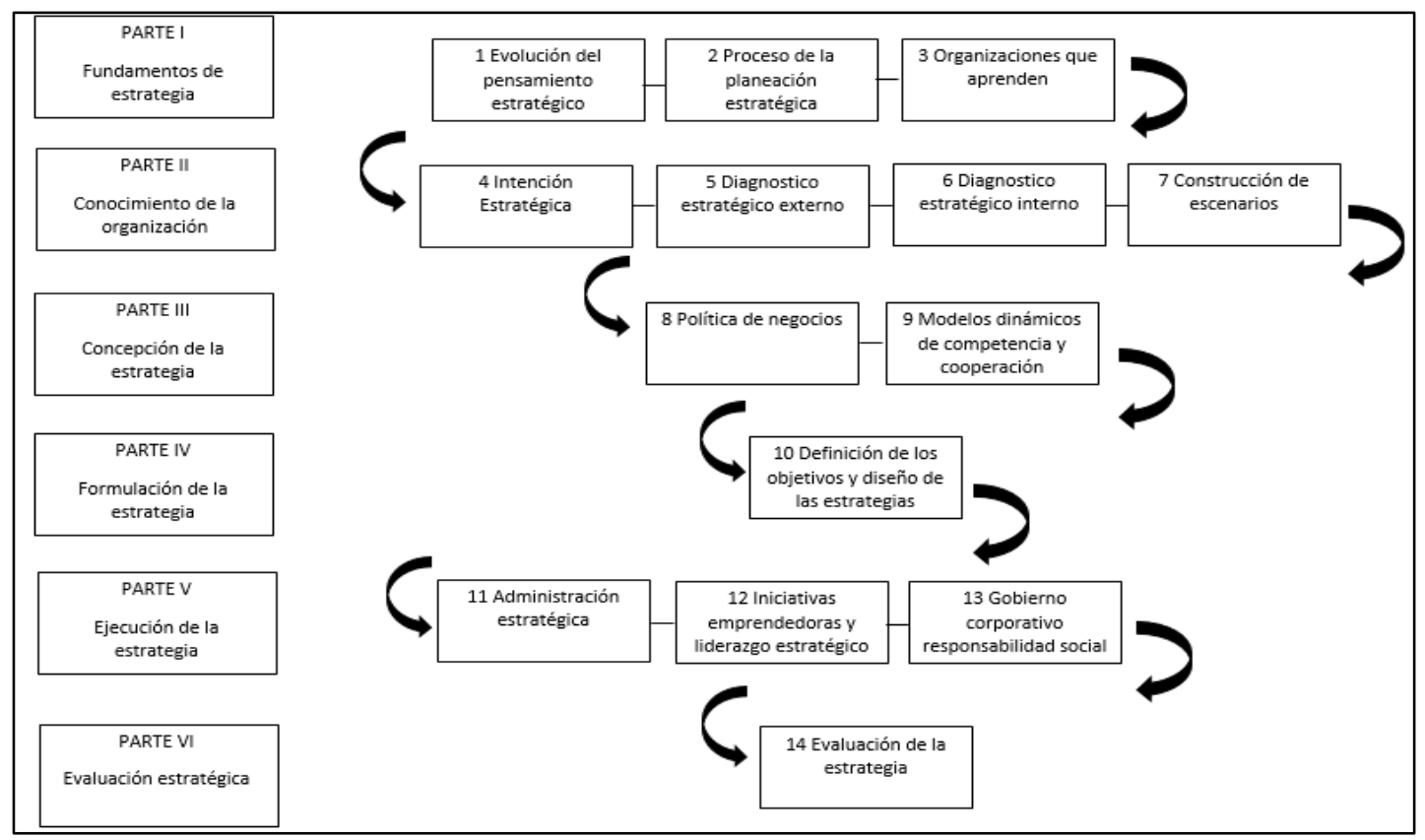

Figura 1. Evaluación de estrategia

Fuente: (Chiavenato \& Sapiro, 2010)

En tal sentido, para posicionar el criterio de esta investigación se realiza el análisis de las aristas mediante la triangulación por referentes similares. (tabla 2.)

Tabla 2. Triangulación de la variable de investigación

\begin{tabular}{lllll}
\hline Descripción & Herramientas de gestión & $\begin{array}{l}\text { Evaluación } \\
\text { estrategias }\end{array}$ & $\begin{array}{l}\text { Análisis } \\
\text { categorial }\end{array}$ \\
\hline Definición & Proporciona & Es una fase de la & Metodologías \\
& metodologías por la & administración & Fases \\
& dirección que facilita el & estratégica, dirigida & Estrategias \\
& control de operación. & por los de la alta & Evaluar \\
& Fundamental & gerencia para evaluar & Dirección \\
& conocimiento de activos & la manera por la cual & Alta gerencia \\
& intangibles & alcanzaron & los \\
& Evalúa las competencias & objetivos & \\
& personales & & Competencia \\
\hline
\end{tabular}




$\begin{array}{lll}\begin{array}{l}\text { Gestión por competencia } \\ \text { Balance Scorecard }\end{array} & \begin{array}{l}\text { Estrategias } \\ \text { Operación } \\ \text { Business }\end{array} & \begin{array}{l}\text { Estrategias } \\ \text { Procedimientos }\end{array} \\ \begin{array}{l}\text { Prospectiva } \\ \text { Outsourcing }\end{array} & \\ \begin{array}{l}\text { Reingeniería } \\ \text { procesos }\end{array} & \text { de } & \\ \text { Empowermentos } & & \\ & & \end{array}$

Elaboración propia a partir de la bibliografía consultada

\section{Materiales y métodos}

Para el diagnóstico de las variables se realizó una revisión documental de los documentos emitidos por el organismo de control, y la información presentada por la entidad regulada a través de: boletines, guías, lineamientos; para proceder a la triangulación de la información investigada.

La revisión documental permite identificar las investigaciones elaboradas con anterioridad, las autorías y sus discusiones; delinear el objeto de estudio; construir premisas de partida; consolidar autores para elaborar una base teórica; hacer relaciones entre trabajos; rastrear preguntas y objetivos de investigación; observar las estéticas de los procedimientos (metodologías de abordaje); establecer semejanzas y diferencias entre los trabajos y las ideas del investigador; categorizar experiencias; distinguir los elementos más abordados con sus esquemas observacionales; y precisar ámbitos no explorados. (Valencia, 2015)

Los Cuerpos de Bomberos al ser parte del sector público están regido bajo la Ley de transparencia emite informes mensuales de los proyectos o programas a ejecutar o ejecutados, a su vez las metas y objetivos alcanzados, sin embargo son escasas las publicaciones en donde se socializan los resultados de verificación del cumplimiento de objetivos en donde se plasme el soporte a la gestión de estas entidades; para el estudio se ha considerado como unidad de análisis al cuerpo de bomberos de Shishufindi por encontrarse asentado en una zona considerada vulnerable, por lo tanto constitucionalmente de atención prioritaria. (tabla 3.)

Tabla 3. Revisión documental de herramientas de gestión de los cuerpos de bomberos

\begin{tabular}{|c|c|c|c|c|c|}
\hline $\begin{array}{c}\text { Unidad de } \\
\text { análisis }\end{array}$ & & Documento & Enfoque & & Análisis categorial \\
\hline $\begin{array}{l}\text { Cuerpo } \\
\text { Bomberos }\end{array}$ & $\begin{array}{l}\text { de } \\
\text { del }\end{array}$ & $\begin{array}{lr}\text { Planes y programas } \\
\text { de en } & \text { ejecución } \\
\text { enero } & 2018 .\end{array}$ & $\begin{array}{l}\text { Informar } \\
\text { programación } \\
\text { planificación }\end{array}$ & $\begin{array}{l}\text { la } \\
y \\
\text { en }\end{array}$ & Talento Humano \\
\hline
\end{tabular}




\begin{tabular}{|c|c|c|c|c|}
\hline $\begin{array}{l}\text { Cantón } \\
\text { Shushufindi }\end{array}$ & & $\begin{array}{l}\text { (Cuerpo de } \\
\text { Bomberos } \\
\text { Shushufindi, 2018) }\end{array}$ & $\begin{array}{l}\text { ejecución del mes } \\
\text { de enero del } 2018\end{array}$ & \\
\hline $\begin{array}{l}\text { Cuerpo } \\
\text { Bomberos } \\
\text { Cantón } \\
\text { Shushufindi }\end{array}$ & $\begin{array}{l}\text { de } \\
\text { del }\end{array}$ & $\begin{array}{lr}\text { Objetivos y } & \text { metas } \\
\text { enero } & 2018 . \\
\text { (Cuerpo } & \text { de } \\
\text { Bomberos } & \\
\text { Shushufindi, 2018) }\end{array}$ & $\begin{array}{l}\text { Informar de cada } \\
\text { unidad el } \\
\text { porcentaje de metas } \\
\text { y objetivos } \\
\text { cumplido del mes } \\
\text { de enero del } 2018\end{array}$ & $\begin{array}{l}\text { Procesos } \\
\text { Gobernantes. } \\
\text { Procesos de } \\
\text { agregadores de } \\
\text { valores: Gestión de } \\
\text { prevención de } \\
\text { incendios, gestión } \\
\text { de especialización } \\
\text { bomberil, gestión } \\
\text { de siniestros. } \\
\text { Nivel de apoyo: } \\
\text { Departamento } \\
\text { Administrativo, } \\
\text { financiero y talento } \\
\text { humano }\end{array}$ \\
\hline $\begin{array}{l}\text { Cuerpo } \\
\text { Bomberos } \\
\text { Cantón } \\
\text { Shushufindi }\end{array}$ & $\begin{array}{l}\text { de } \\
\text { del }\end{array}$ & $\begin{array}{l}\text { Planes y programas } \\
\text { de en ejecución } \\
\text { febrero } \\
\text { (Cuerpo } \\
\text { Bomberos } \\
\text { Shushufindi, 2018) }\end{array}$ & $\begin{array}{lr}\text { Informar } & \text { la } \\
\text { programación } & \text { y } \\
\text { planificación } & \text { en } \\
\text { ejecución del mes } \\
\text { de febrero del } 2018\end{array}$ & Talento Humano \\
\hline $\begin{array}{l}\text { Cuerpo } \\
\text { Bomberos } \\
\text { Cantón } \\
\text { Shushufindi }\end{array}$ & $\begin{array}{l}\text { de } \\
\text { del }\end{array}$ & $\begin{array}{lr}\text { Objetivos y } & \text { metas } \\
\text { febrero } & 2018 . \\
\text { (Cuerpo } & \text { de } \\
\text { Bomberos } & \\
\text { Shushufindi, 2018) }\end{array}$ & $\begin{array}{lr}\text { Informar de } & \text { cada } \\
\text { unidad } & \text { el } \\
\text { porcentaje de metas } \\
\text { y } \\
\text { cumplido del mes } \\
\text { de febrero del } 2018\end{array}$ & $\begin{array}{l}\text { Procesos } \\
\text { Gobernantes. } \\
\text { Procesos de } \\
\text { agregadores de } \\
\text { valores: Gestión de } \\
\text { prevención de } \\
\text { incendios, gestión } \\
\text { de especialización } \\
\text { bomberil, gestión } \\
\text { de siniestros. } \\
\text { Nivel de apoyo: } \\
\text { Departamento } \\
\text { Administrativo, } \\
\text { financiero y talento } \\
\text { humano }\end{array}$ \\
\hline
\end{tabular}




\begin{tabular}{|c|c|c|c|c|}
\hline $\begin{array}{l}\text { Cuerpo } \\
\text { Bomberos } \\
\text { Cantón } \\
\text { Shushufindi }\end{array}$ & $\begin{array}{l}\text { de } \\
\text { del }\end{array}$ & $\begin{array}{lr}\text { Planes y programas } \\
\text { de en ejecución } \\
\text { marzo } & 2018 . \\
\text { (Cuerpo } & \text { de } \\
\text { Bomberos } & \\
\text { Shushufindi, 2018) }\end{array}$ & $\begin{array}{l}\text { Informar la } \\
\text { programación } \\
\text { planificación en } \\
\text { ejecución del mes } \\
\text { de marzo del } 2018\end{array}$ & Talento Humano \\
\hline $\begin{array}{l}\text { Cuerpo } \\
\text { Bomberos } \\
\text { Cantón } \\
\text { Shushufindi }\end{array}$ & $\begin{array}{l}\mathrm{de} \\
\mathrm{del}\end{array}$ & 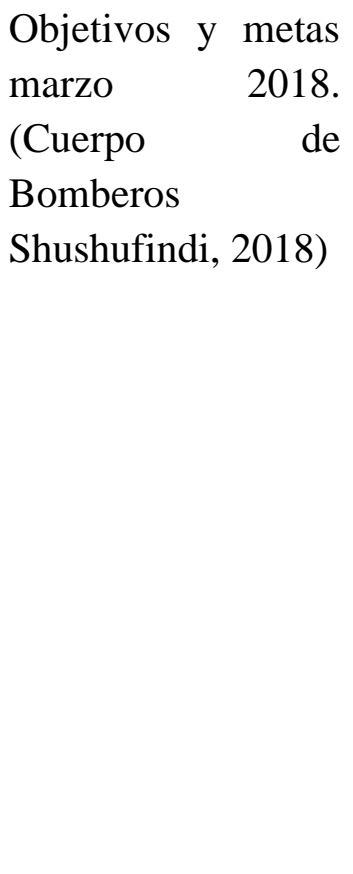 & $\begin{array}{l}\text { Informar de cada } \\
\text { unidad } \\
\text { porcentaje de metas } \\
\text { y objetivos } \\
\text { cumplido del mes } \\
\text { de marzo del } 2018\end{array}$ & $\begin{array}{l}\text { Procesos } \\
\text { Gobernantes. } \\
\text { Procesos de } \\
\text { agregadores de } \\
\text { valores: Gestión de } \\
\text { prevención de } \\
\text { incendios, gestión } \\
\text { de especialización } \\
\text { bomberil, gestión } \\
\text { de siniestros. } \\
\text { Nivel de apoyo: } \\
\text { Departamento } \\
\text { Administrativo, } \\
\text { financiero y talento } \\
\text { humano }\end{array}$ \\
\hline $\begin{array}{l}\text { Cuerpo } \\
\text { Bomberos } \\
\text { Cantón } \\
\text { Shushufindi }\end{array}$ & $\begin{array}{l}\text { de } \\
\text { del }\end{array}$ & $\begin{array}{l}\text { Planes y programas } \\
\text { de en ejecución } \\
\text { abril } 2018 . \text { (Cuerpo } \\
\text { de } \quad \text { Bomberos } \\
\text { Shushufindi, 2018) }\end{array}$ & $\begin{array}{lr}\text { Informar } & \text { la } \\
\text { programación } & \text { y } \\
\text { planificación en } \\
\text { ejecución del mes } \\
\text { de abril del } 2018\end{array}$ & Talento Humano \\
\hline $\begin{array}{l}\text { Cuerpo } \\
\text { Bomberos } \\
\text { Cantón } \\
\text { Shushufindi }\end{array}$ & $\begin{array}{l}\text { de } \\
\text { del }\end{array}$ & $\begin{array}{l}\text { Objetivos y metas } \\
\text { abril 2018. (Cuerpo } \\
\text { de } \quad \text { Bomberos } \\
\text { Shushufindi, 2018) }\end{array}$ & $\begin{array}{l}\text { Informar de cada } \\
\text { unidad } \\
\text { porcentaje de metas } \\
\text { y objetivos } \\
\text { cumplido del mes } \\
\text { de abril del } 2018\end{array}$ & $\begin{array}{l}\text { Procesos } \\
\text { Gobernantes. } \\
\text { Procesos de } \\
\text { agregadores de } \\
\text { valores: Gestión de } \\
\text { prevención de } \\
\text { incendios, gestión } \\
\text { de especialización } \\
\text { bomberil, gestión } \\
\text { de siniestros. } \\
\text { Nivel de apoyo: } \\
\text { Departamento } \\
\text { Administrativo, }\end{array}$ \\
\hline
\end{tabular}


financiero y talento

humano

$\begin{array}{lrllrl}\text { Cuerpo } & \text { de } & \text { Planes y programas } & \text { Informar } & \text { la } & \text { Talento Humano } \\ \text { Bomberos } & \text { del } & \text { de en ejecución } & \text { programación } & \text { y } \\ \text { Cantón } & & \text { mayo } & 2018 . & \text { planificación en } & \\ \text { Shushufindi } & & \text { (Cuerpo } & \text { de } & \text { ejecución del mes } & \\ & & \text { Bomberos } & & \text { de mayo del } 2018\end{array}$

Shushufindi, 2018)

Cuerpo

Bomberos

Cantón

Shushufindi de Objetivos y metas

del mayo

(Cuerpo

Bomberos

Shushufindi, 2018)

2018.
de

Informar de cada unidad porcentaje de metas y objetivos cumplido del mes de mayo del 2018 el Gobernantes.

Procesos de agregadores de valores: Gestión de prevención de incendios, gestión de especialización bomberil, gestión de siniestros.

Nivel de apoyo: Departamento Administrativo, financiero y talento humano

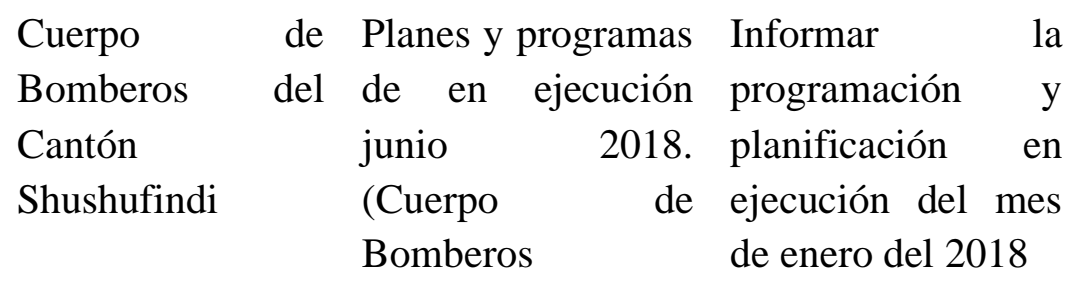

Shushufindi, 2018)

$\begin{array}{lrlrlr}\text { Cuerpo } & \text { de } & \text { Objetivos y } & \text { metas } & \text { Informar de cada } \\ \text { Bomberos } & \text { del } & \text { junio } & 2018 . & \text { unidad r } \\ \text { Cantón } & - & \text { (Cuerpo } & \text { de } & \text { porcentaje de metas } \\ \text { Shushufindi } & & \text { Bomberos } & & \text { y } & \text { objetivos } \\ & & \text { Shushufindi, 2018) } & \text { cumplido del mes } \\ & & & \text { de junio del 2018 }\end{array}$

Procesos

Gobernantes.

Procesos de agregadores de valores: Gestión de prevención de incendios, gestión de especialización bomberil, gestión de siniestros. 
Nivel de apoyo:

Departamento

Administrativo,

financiero y talento

humano

Fuente: (Cuerpo de Bomberos Shushufindi, 2018)

Elaboración: propia a partir de la bibliografía consultada

El GAD al ser el organismo de control realiza su trabajo de control sin embargo carecen de reportes referentes a la gestión que realiza el Cuerpo de Bomberos; a pesar de que al ser una unidad adscrita del GAD Municipal debe periódicamente informar sobre la gestión que están efectuando, y a su vez controlar los planes y programas que se ejecutan y están por ejecutarse del mismo. (tabla 4.)

Tabla 4. Revisión documental de reportes emitidos por el GAD Municipal Shushufindi

\begin{tabular}{|c|c|c|c|}
\hline $\begin{array}{l}\text { Organismo de } \\
\text { control }\end{array}$ & Documento & Enfoque & $\begin{array}{l}\text { Análisis } \\
\text { categorial }\end{array}$ \\
\hline $\begin{array}{l}\text { GAD Municipal } \\
\text { de Shushufindi }\end{array}$ & $\begin{array}{l}\text { BOLETÍN } \quad \mathrm{N}^{\circ}: \\
\text { 000021-GADMSFD- } \\
2018\end{array}$ & $\begin{array}{l}\text { Las unidades adscritas } \\
\text { que también } \\
\text { transparentaron su } \\
\text { trabajo fueron: } \\
\text { Registro de la } \\
\text { Propiedad, Cuerpo de } \\
\text { Bomberos del Cantón } \\
\text { Shushufindi }\end{array}$ & $\begin{array}{l}\text { Transparentacion } \\
\text { información }\end{array}$ \\
\hline
\end{tabular}

Fuente: (Gobierno Autónomo Desentralizado Municipal de Shushufindi, 2018)

Elaboración: propia a partir de la bibliografía consultada

El GAD deberá tomar un conjunto de decisiones y acciones de directivas, las cuales deben estar alineados a las normativas y valores de la institución basado en un correcto manejo de las herramientas que ayude verificarlas estrategias establecidas para cumplir con lo planteados, esto deben realizarse de manera continua, y no al final de periodos específicos o justo después de que los problemas ocurren.

\section{Resultados y discusión}

Una vez investigadas las variables, realizada la revisión documental de reportes emitidos por los organismos de control, se realizó la triangulación de información y se obtuvo el resultado que los organismos de control no cuentan con una herramienta de gestión para evaluar las estrategias planteadas por el cuerpo de bomberos, por lo que en esta investigación se estructurará herramientas de gestión basadas en el proceso administrativo. El proceso básico de control comienza con el establecimiento de los 
objetivos o estándares de desempeño, de ahí se evalúa o se mide el desempeño actual, comparando el desempeño actual con los objetivos o los estándares establecidos, para así tomar medidas correctivas para enmendar las posibles debilidades.

Para ello se debe fundamentar la estrategia, conocer acerca de la organización, concepción y formulación de la estrategia, ejecución de la estrategia y evaluar la estrategia realizada y se plantea el modelo conceptual de herramientas de gestión y evaluación de estrategias. El modelo conceptual, como representación de los componentes y las relaciones que se presentan para un fenómeno Galarza (2007), Almuiñas \& Galarza (2010, 2011), Ferriol (2011).

Se reconocen como aspectos referidos al diseño del modelo, los que tratan Galarza (2007), Almuiñas \& Galarza (2010, 2011), Ferriol (2011); Mayorga, Álvarez, Mayorga \& Herrera (2018); Mayorga, Quezada, Álvarez, Alvarado, López, \& López (2018): 1. Componentes del modelo, se definen como las variables fundamentales de herramientas de gestión y evaluación de estrategias; 2. Los elementos, que se definen como las dimensiones de las variables: a. metodología, proceso, revisión b. establecimiento de estrategias, comparación del desempeño actual con los objetivos; 3. Las "fronteras", que establecen los términos y límites en que actúa la organización estudiada, los que se consideran apoyo para el desarrollo de la concepción de integración planteada: a. Marco jurídico vigente; b. Organismo rector de control y fiscalización, se reconoce a las Gobiernos Autónomos Descentralizados Municipales o Metropolitano que a través de la unidad de fiscalización viabiliza la aplicación de procedimientos coherentes con la necesidad nacional; 4. El resultado de las interacciones del modelo propuesto constituye el informe de evaluación de estrategia para la toma de medidas correctivas. (figura 2).

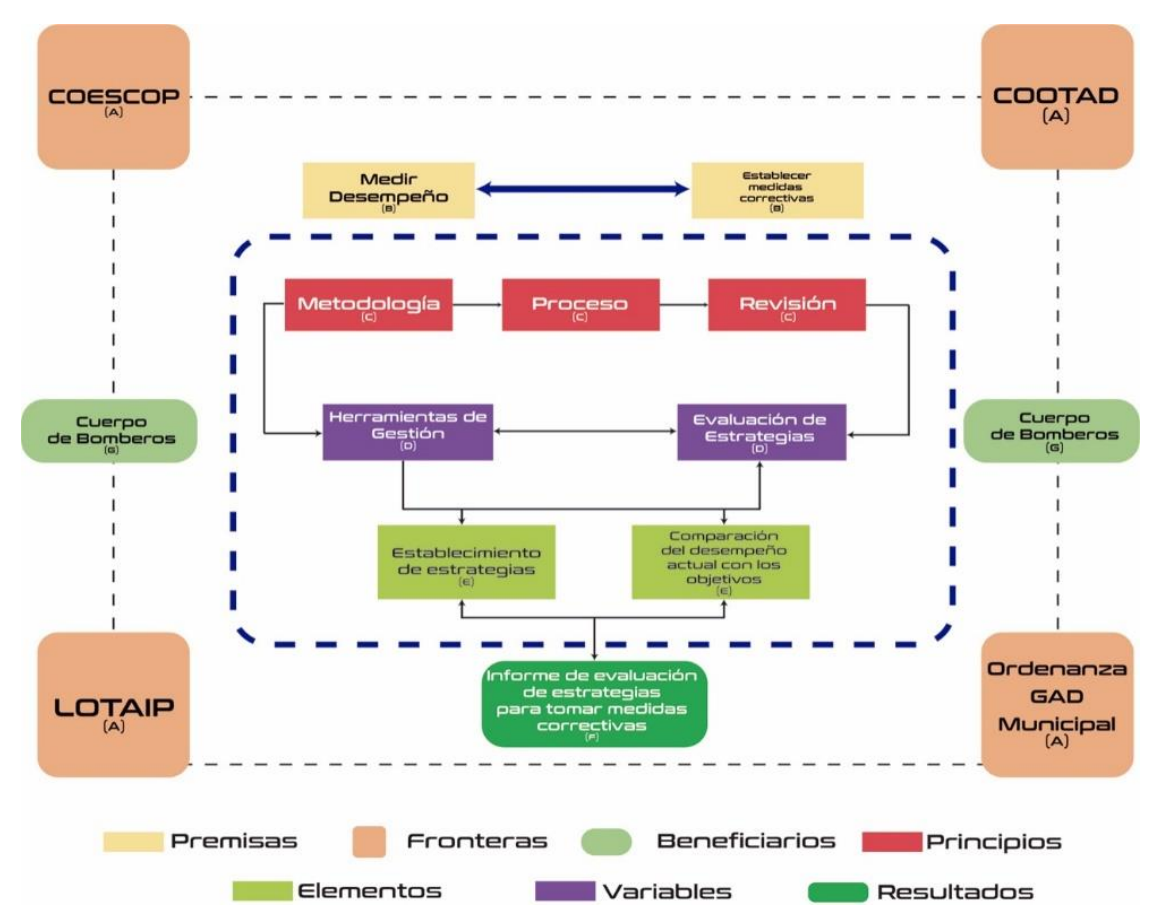

Figura 2. Modelo conceptual de herramientas de gestión y evaluación de estrategias Elaboración propia 


\section{Conclusiones}

- Los organismos de control no cuentan con una herramienta de gestión para evaluar las estrategias utilizadas por las instituciones adscritas a ellas; es decir que los GAD Municipales o metropolitanos no tiene el debido control a los Cuerpos de Bomberos, para lo cual el modelo conceptual propuesto ayudara para que los organismos de control evalúan de manera correcta y las instituciones adscritas puedan realizar medidas correctivas apropiadas

\section{Bibliografía}

Angulo, R. (2017). Gestión del conocimiento y aprendizaje organizacional: una visión integral. Revista Universidad Pontificia Bolivariana, 17(1), 53-70. Recuperado el 13 mayo de 2019, de https://revistas.upb.edu.co/index.php/informespsicologicos/article/view/7350/67 10

Aranda, M., Ordoñez, L., \& Peralta, C. (2018). La gestión por procesos como medio para mejorar la eficacia en el cumplimiento de objetivos institucionales del Minagri. Recuperado el 05 de septiembre de 2019, de http://repositorio.up.edu.pe/bitstream/handle/11354/2270/Merly_Tesis_maestria _2018.pdf?sequence=1\&isAllowed=y

Bolaños, E. L. (2016). La gestión de la calidad en Perú: un estudio de la norma ISO 9001, sus beneficios y los principales cambios en la versión 2015. Revista Universidad \& Empresa, 18(30), 33-54. Recuperado el 13 de mayo de 2019, de http://www.redalyc.org/pdf/1872/187244133006.pdf

Carvajal, A., \& Escobar, M. (2013). Herramienta integrada de control interno y administrador de riesgos, enmarcada en un buen Gobierno Corporativo para pequeñas y medianas empresas en Colombia. Apuntes Contables(16), 19-37. Recuperado el 05 de septiembre de 2019, de https://revistas.uexternado.edu.co/index.php/contad/article/view/3357/3007

Casanova, R. (20 de julio de 2010). roberto-casanova.blogspot.com. Recuperado el 25 de mayo de 2019, de https://roberto-casanova.blogspot.com/2010/07/historia-de-losbomberos-en-el-mundo.html

Chiavenato, A., \& Sapiro, A. (2010). Planeación estrategica. Fundamentos y aplicaciones. Mexico, D. F, Mexico: Editorial Mc Graw Hill.

Código Orgánico de las Entidades de Seguridad Ciudadana y Orden Público COESCOP. (2017). Subseccion III CUERPOS DE BOMBEROS art. 276 Funciones. Refistro oficial 19. Ecuador. 
Código Orgánico de las Entidades de Seguridad Ciudadana y Orden Público COESCOP. (2017). Subsección III CUERPOS DE BOMBEROS art. 285 Modelo de gestión cantonal. Registro oficial 19. Ecuador.

Código Orgánico de Organización Territorial, Autonomía y Descentralización COOTAD. (2010). Art.3 Principios. Registro oficial 303. Ecuador.

Consititucion del Ecuador. (2008). Art.11 EI ejercicio de los derechos. Registro oficial 449. Ecuador.

Constitución del Ecuador. (2008). Art. 226 Las instituciones del Estado. Registro oficial 449. Ecuador.

Cruz, A. (2003). La evaluación de la estrategia empresarial. Asociación Cubana de Producción Anima(1), 52-54. Recuperado el 05 de septiembre de 2019, de http://www.actaf.co.cu/revistas/Revista\%20ACPA/2003/REVISTA\%2001/17\% 20LA\%20EVALUACION.pdf?fbclid=IwAR2POSFgvSW26X_j7WPIDwGhyz0 zl2bVmHl8fi0sHCwwpcUgpAHI6K-yYyo

Cruz, A. (2006). La planificación estrategica. Recuperado el 14 de mayo de 2019, de http://www.contraloriageneralzulia.org.ve/articulos/LA_PLANIFICACION_ES TRATEGICA_02.pdf.

Cuatrecasa, L., \& Gonzales, J. (2017). Gestión integral de la calidad. Barcelona: Profit Editorial.

Cuerpo de Bomberos Shushufindi. (2018). https://bomberosshushufindi.gob.ec/hope/. Recuperado el 14 de mayo de 2019, de https://bomberosshushufindi.gob.ec/hope/index.php/transparencia/ley-detransparencia/category/1-lotaip-2018

Cuerpo de bomberos voluntaio amaga. (28 de agosto de 2018). bomberosamaga.blogspot.com. Recuperado el 25 de mayo de 2019, de http://bomberosamaga.blogspot.com/

Educaweb. (s.f.). www.educaweb.com. Recuperado el 25 de mayo de 2019, de https://www.educaweb.com/profesion/bombero-97/

Fred, D., \& Forest, D. (2017). Conceptos de administración estrategica. Mexico, D. F, Mexico: Pearson Education de Mexico S.A de C.V.

Gobierno Autónomo Descentralizado Municipal de Shushufindi. (19 de 05 de 2016). shushufindi.gob.ec. Recuperado el 05 de junio de 2019, de http://shushufindi.gob.ec/portal/concejo-municipal/ordenanzas/

Gobierno Autónomo Desentralizado Municipal de Shushufindi. (03 de abril de 2018). www.shushufindi.gob.ec. Recuperado el 14 de mayo de 2019, de http://shushufindi.gob.ec/portal/wpcontent/uploads/2018/06/BOLETI\%CC\%81N-0021-RENDICIO\%CC\%81NDE-CUENTAS.pdf

Gonzáles, A., \& Tapia, N. (2016). Gestión del talento humano: Herramienta clave para el desarrollo organizacional (Estudio en las empresas comercializadoras de 
electrodomésticos). Revista Observatorio de la Economía Latinoamericana., 111. Recuperado el 13 de mayo de 2019, de http://www.eumed.net/cursecon/ecolat/ec/2016/crecimiento.html

Henao, O., Quintero, N., Ramirez, D., Aálvarez, D., Gomez, P., Otalvaro, C., \& Bronacelly, J. (2016). Planes de lectura y escritura, valoraciones en torno al cumplimiento de objetivos y apropiaciones: el caso del plan de lectura y escritura de Medellín, 2009-2014. Revista de ciencias de la información, bibliotecnología $y$ archivista(2), 9-41. Recuperado el 05 de septiembre de 2019, de https://cnb.gov.co/codices/online/Vol12-2016II.pdf\#page $=98$

Hernandes, D., \& Escobar, A. (2017). Modelo de contabilidad social como herramienta de gestión para la responsabilidad social empresarial. IJMSOR: International Journal of Management Science \& Operation Research, 2(1), 44-56. Obtenido de http://ijmsoridi.com/index.php/ijmsor/article/view/86

Hernandez, R. (2014). Metodología de la investigación. Mexico, D F, Mexico: Editorial Mc Graw Hill.

Ley Orgánica de Transparencia y Acceso a la Información Pública LOTAIP. (2004). Art. 7 Difusion de la infomación pública. Registro oficial 337. Ecuador.

Mayorga, M., Álvarez, G., Mayorga, L., \& Herrera, K. (2018). Auditoría de gestión con un enfoque de responsabilidad social. Quito: Jurídica del Ecuador.

Mayorga-Díaz, M. P., Quezada-Sarmiento, P. A., Álvarez-Gómez, G., AlvaradoCamacho, P. E., López-Jara, A. A., \& Lopez-Paredes, A. (2018, June). Conceptual model and process applied in management auditic with focus in social responsibility through open source tools. In 2018 13th Iberian Conference on Information Systems and Technologies (CISTI) (pp. 1-6). IEEE.

Medina, D., Medina, A., \& Nogueira, D. (2017). Procesos y factores calves de la gestión del conocimiento. Revista Universidad y sociedad, 9(3), 15-23. Recuperado el 13 de mayo de 2019, de http://scielo.sld.cu/pdf/rus/v9n3/rus02317.pdf

Mendez, C. (2009). Tecnologias y herramientas de gestión. Caso: grandes medianas y pequeñas empresas en Bogota. Bogota, Colombia: Editorial Universidad del Rosario. Recuperado el 13 de mayo de 2019, de https://books.google.com.ec/books?id=_3wKbb40aAgC\&printsec=frontcover\&s ource $=$ gbs_ge_summary_r $\&$ cad $=0 \# v=$ onepage $\& \mathrm{q} \& \mathrm{f}=$ false

Mora, C. (2015). El buen gobierno corporativo y su incidencia en el cumplimiento de objetivos y nivel de desempeño de las Cajas Municipales de Ahorro y Crédito. Trujillo.

Mora, L., Duran, M., \& Zambrano, J. (2016). Consideraciones actuales sobre gestión empresarial. Revista Cientifica Dominio de la Ciencia, 2(4), 511-520. Recuperado el 13 de mayo de 2019, de https://dialnet.unirioja.es/servlet/articulo?codigo $=5802891$

Muñoz, A. (1999). La gestión de la calidad total en la administración pública. Madrid, España: Editorial Diaz de Santos S.A. Recuperado el 13 de mayo de 2019 
Opazo, M., Sépulveda, A., \& Perez, M. (2015). Estrategias de evaluación del aprendizaje en la universidad y tareas auténticas: percepción de los estudiantes. Dialogos Educativos, 19-33. Obtenido de https://dialnet.unirioja.es/descarga/articulo/5159507.pdf

Palma, V., \& Burgos, J. (2016). La auditoría integral: un enfoque hacia el cumplimiento de los objetivos corporativos en las empresas. Revista Caribeña de Ciencias Sociales. Obtenido de www.eumed.net/rev/caribe/2016/09/objetivos.zip

Pereira, C. (2019). Actualidad de la gestión empresarial en las pymes. Apuntes Contables(24), 39-53. Recuperado el 05 de septiembre de 2019, de https://revistas.uexternado.edu.co/index.php/contad/article/view/5982/7704

Plan Nacional de Desarrollo. (2017). Objetivo 5. Impulsar la productividad y competitividad para el crecimiento económico sostenible de manera redistributiva y solidaria. Ecuador.

Rodriguez, J. (2003). Introducción a la administración con enfoque a de sistemas. Mexico: Editorial Thompson. Recuperado el 13 de mayo de 2019

Secretaría de gestión de riesgo. (2016). www.gestionderiesgos.gob.ec. Recuperado el 05 de septiembre de 2019, de https://www.gestionderiesgos.gob.ec/wpcontent/uploads/downloads/2016/03/gu\%C3\%ADa-operativa-organizacionalcuerpo-de-bomberos.pdf

Secretaría de gestión de riesgo. (2017). www.gestionderiesgos.gob.ec. Recuperado el 05 de septiembre de 2019, de https://www.gestionderiesgos.gob.ec/wpcontent/uploads/downloads/2018/02/Proyecto-Fortalecimiento-EquipamientoCuerpos-de-Bomberos.pdf

Valencia, V. (2015). Revisión documental en el proceso de investigación. Universidad Tecnológica de Pereira. Recuperado el 20 de 08 de 2019, de https://univirtual.utp.edu.co/pandora/recursos/1000/1771/1771.pdf

Vargas, M., \& Aldana, L. (2014). Calidad y servicio conceptos y herramientas. Bogota: Ecoe ediciones.

Velazco, J. P. (2009). Gestion por procesos. Madrid, España: ESIC Editorial. Recuperado el 13 de mayo de 2019, de https://books.google.com.ec/books?id=koSkh64nRb4C\&printsec=frontcover\&so urce $=\mathrm{gbs} \_g e \_s u m m a r y \_r \& c a d=0 \# \mathrm{v}=$ onepage $\& \mathrm{q} \& \mathrm{f}=\mathrm{false}$

Villamizar, A. (2011). Gerencia y control del conocimiento en las instituciones universitarias. Apuntes Contables(15), 131-161. Recuperado el 05 de septiembre de 2019 , de https://revistas.uexternado.edu.co/index.php/contad/article/view/2943/2587

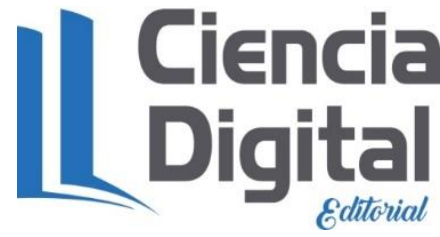




\section{PARA CITAR EL ARTÍCULO INDEXADO.}

Montoya Santillán, M. L., Masaquiza Sailema, K. J., Espinosa Jaramillo, M. T., \& Mayorga Díaz , M. P. (2021). Herramientas de gestión para la evaluación de estrategias en los cuerpos de bomberos. Visionario Digital, 5(2), 70-88. https://doi.org/10.33262/visionariodigital.v5i2.1637

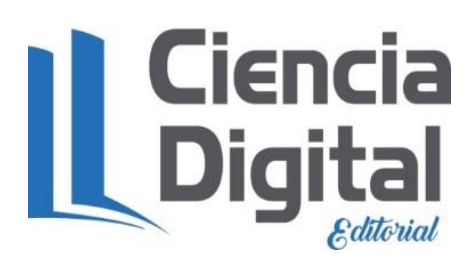

El artículo que se publica es de exclusiva responsabilidad de los autores y no necesariamente reflejan el pensamiento de la Revista Visionario Digital.

El artículo queda en propiedad de la revista y, por tanto, su publicación parcial y/o total en otro medio tiene que ser autorizado por el director de la Revista Visionario Digital.
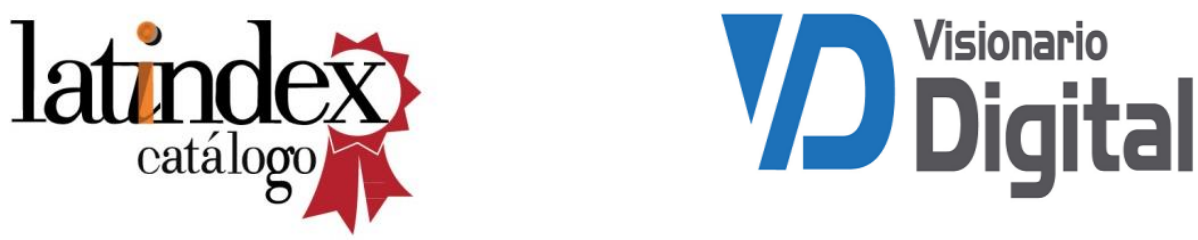